\title{
Coupling Microwave-Assisted Drying and Supercritical Carbon Dioxide Extraction for Coconut Oil Processing
}

\author{
Armando T. Quitain ${ }^{1, *}$, Takashi Moriyoshi ${ }^{2}$, Motonobu Goto ${ }^{3}$ \\ ${ }^{1}$ Graduate School of Science and Technology, Kumamoto University, Kumamoto, Japan \\ ${ }^{2}$ Research Center for Industrial Science and Technology (RIST Kagawa), Kagawa, Japan \\ ${ }^{3}$ Department of Chemical Engineering, Nagoya University, Nagoya, Japan \\ *Corresponding author: quitain@kumamoto-u.ac.jp
}

Received January 01, 2013; Revised February 05, 2013; Accepted February 28, 2013

\begin{abstract}
Supercritical carbon dioxide extraction was applied to the processing of virgin coconut oil (VCNO), which has been known to possess several functions such as antimicrobial properties as proven by many medical doctors and clinical researchers. Prior to extraction, the sample was dried using microwave-assisted low-temperature air drying method. Using these techniques, an oil recovery of $95 \%$ was obtained at $40^{\circ} \mathrm{C}$ and $20 \mathrm{MPa}$ at a processing time of less than $20 \mathrm{~h}$. This is more efficient than the conventional cold-press method which has a normal oil recovery of about 30 to $50 \%$ at a longer processing time of 48 to $72 \mathrm{~h}$. The obtained oil has physical and chemical properties that are almost identical to or better than the commercial VCNO, thus deemed to possess the same antimicrobial and nutritional properties. The effects of extraction conditions were also investigated for optimization analyses.
\end{abstract}

Keywords: supercritical carbon dioxide, extraction, natural product, microwave, virgin coconut oil

\section{Introduction}

Virgin coconut oil (VCNO), which is currently being marketed as a nutritional supplement, is known to provide health benefits like protection from viruses, fungi and bacteria because of its high lauric acid content. Lauric acid is a medium chain fatty acid, and in human or animal body, it naturally transforms into monolaurin, a major component of mother's milk that enhances the immune system of infants [1,2]. The VCNO's unique and abundant antimicrobial properties make it superior to any other oils [3]. One of the many clinical evidences of its therapeutic power is the significant reduction in viral loads of an HIVpositive patient, attributed to the inclusion of coconut in his diet $[3,4,5]$.

To date, extraction of VCNO is mostly done in a community level using a cold-press method but the recovery is low at about 35 to $50 \%$. As the demand for this superior oil increases, Dr. Fife, dubbed as "Dr. Coconut" due to his numerous studies on nutritional and therapeutic properties of $\mathrm{VCNO}$, has urged producers to develop techniques for extraction of VCNO from community level to a standardized continuous production [3].

One promising technique to obtain the oil at low temperature is by supercritical carbon dioxide extraction. This is the most widely used supercritical fluid, mainly because it is cheap, relatively nontoxic, and has convenient critical temperature of $31.1^{\circ} \mathrm{C}$ and critical pressure of 7.4MPa [6]. Supercritical carbon dioxide extraction had been applied in our previous works on the recovery of useful compounds from natural products $[7,8,9,10]$. It has also proven its usefulness as a replacement for organic solvents with its application in large-scale extractions such as coffee decaffeination. Most recently, several plants have been constructed worldwide for the extraction of phytochemicals and nutraceuticals from natural products such as ginseng [11]. In the presence of modifier such as ethanol (EtOH), supercritical carbon dioxide could enhance extraction of polar compounds like polyphenols [12], thus increasing the value and in most cases, the therapeutic property of the extracts. Other technical advantages of supercritical carbon dioxide extraction include higher diffusion coefficient, lower viscosity and absence of surface tension, thus allowing rapid penetration into the pores of heterogeneous matrices, and selectivity during extraction may be manipulated. This technique does not leave chemical residue in the products and reuse of carbon dioxide is also possible $[13,14,15]$.

In the processing of virgin coconut oil, the use of supercritical carbon dioxide has added benefits for easy process standardization, because of tunable operating parameters unlike the complex and tedious conventional cold-press method. Moreover, the quality of the products can also be highly guaranteed because it is a closed system. However, the water content of the coconut may hinder the extraction, and could lower the yield of oil, thus in this regard a microwave-assisted low-temperature air-drying method can be applied to dry the sample prior to extraction.

This study investigated the application of supercritical carbon dioxide to the extraction of virgin coconut oil. To increase extractability of the oil, the sample was pre-dried 
using microwave-assisted low-temperature air drying. The effects of extraction conditions were also investigated for optimization analyses.

\section{Experimental}

\subsection{Coconut Sample}

The coconut sample was obtained from the Philippines and was brought to Japan in unopened shell. The juice was removed from the freshly opened sample, and the meat was grated using a vegetable slicer. The water content of about $50 \mathrm{wt} \%$ of the grated coconut was then removed by either microwave-assisted hot-air drying at $40^{\circ} \mathrm{C}$ or freeze-drying. The dried sample contains an oil fraction of about $65 \mathrm{wt} \%$, as determined by Soxhlet extraction with hexane for $5 \mathrm{~h}$. This agrees with the data reported for copra, a solar-dried coconut meat [16]. This percentage of oil was taken as the basis for the calculation of the oil recovery in the succeeding supercritical carbon dioxide extraction experiments.

\subsection{Microwave-Assisted Low-Temperature Air Drying}

Coconut samples were dried using a microwaveassisted hot-air drying apparatus (Shikoku Instrumentation Co. Ltd., Japan). The apparatus operates at a frequency of $2.45 \mathrm{GHz}$, similar to that of a domestic microwave oven, with a power programmable from 0 to $1500 \mathrm{~W}$. Hot air at $40^{\circ} \mathrm{C}$ was circulated, while microwave was irradiated to raise the temperature of the sample, while controlling it at $40^{\circ} \mathrm{C}$. In a typical drying experiment, about $1 \mathrm{~kg}$ of coconut sample was placed inside the microwave cavity, then was dried until almost all the water content was removed, while measuring the changes in the weight of the sample every hour.

\subsection{Supercritical $\mathrm{CO}_{2}$ Extraction}

Extraction experiments on coconut samples at pressures up to $20 \mathrm{MPa}$ were carried out using an experimental apparatus shown schematically in Figure 1. The typical operating temperature was set at $40^{\circ} \mathrm{C}$ and the pressure at $20 \mathrm{MPa}$. In optimization studies, the temperature and pressure were varied in the range of 28 to $40^{\circ} \mathrm{C}$ and 7 to $30 \mathrm{MPa}$, respectively. Experiments at a pressure of $30 \mathrm{MPa}$ were done in another apparatus almost similar to the one in the figure except that carbon dioxide was introduced from the bottom of the extractor, passing through the sample in an upward direction.

In a typical run, about $70 \mathrm{~g}$ of dried coconut meat sample was placed in a 300-ml extractor (OM Labtech Co., Ltd, Japan). The extractor temperature was gradually set to the desired level, while pumping carbon dioxide (purity of $99.5 \%$ ) into the extractor to reach a system pressure of $20 \mathrm{MPa}$ using a high-pressure pump (NS personal pump, NP-KX-500J, Nihon Seimitsu Kagaku Co., Ltd., Japan). The system was held for $2 \mathrm{~h}$ at desired conditions, then carbon dioxide was allowed to flow continuously through the extractor for $5 \mathrm{~h}$ at a rate of about $2 \mathrm{~L} / \mathrm{min}$ at room temperature. The extracts were collected in a flask immersed in an iced water bath at around $5^{\circ} \mathrm{C}$.
In ethanol-modified supercritical carbon dioxide experiments, after reaching a pressure of $8 \mathrm{MPa}$, ethanol (Wako Chemicals, 99.5\%) was added gradually using a JASCO Intelligent HPLC pump (PU-980) to reach the desired concentration.

\subsection{High Performance Liquid Chromatography (HPLC) for Analysis of Triglycerides}

The chemical compositions of the oil samples were analyzed using a JASCO Intelligent HPLC-RI apparatus. A mixture of acetonitrile, tetrahydrofuran and dichloromethane at 70, 20 and $10 \%(\mathrm{v} / \mathrm{v})$, respectively served as the eluent at a flow rate of $1.0 \mathrm{ml} / \mathrm{min}$. The column temperature was set constant at $40^{\circ} \mathrm{C}$ throughout the analysis. The injection volume was $10 \mu \mathrm{l}$. Component separation was carried out in an Inertsil ODS-3V column (250 x $4.6 \mathrm{~mm})$, and component detection was performed using an RI detector.

\subsection{Gas Chromatography-Flame Ionization Detector (GC-FID) for Analysis of Fatty Acid Contents}

An improved rapid method developed by Ichihara et al. [17] was applied to determine the fatty acid contents of the oil. About $10 \mathrm{mg}$ of the sample was dissolved in $2 \mathrm{ml}$ hexane (HPLC grade, Wako Chemicals), followed by the addition of $0.2 \mathrm{ml}$ of $2 \mathrm{M}$ methanolic $\mathrm{KOH}$. The tube was vortexed for $2 \mathrm{~min}$ at room temperature. After mild centrifugation at $2000 \mathrm{G}$ for about 2 min using Chibitan-R centrifuge (Millipore, Japan), an aliquot portion of the hexane layer was collected for GC-FID analysis.

Analysis was carried out using a Shimadzu GC-FID (GC-14B) analytical apparatus. The column was a Chrompack capillary column (CP Sil x88 Tailor Made FAME, $0.25 \mathrm{~mm} \times 50 \mathrm{~m}$ ) from GL Sciences Co. (Japan). The injector and the auxiliary temperatures were set at 220 and $250^{\circ} \mathrm{C}$, respectively. The column temperature was initially set at $100^{\circ} \mathrm{C}$ for $4 \mathrm{~min}$, and allowed to ramp up to $210^{\circ} \mathrm{C}$ at a rate of $10^{\circ} \mathrm{C} / \mathrm{min}$, and then was maintained at $210^{\circ} \mathrm{C}$ for $5 \mathrm{~min}$. The carrier gas $(\mathrm{He}, 99.99 \%)$ pressure was $0.2 \mathrm{MPa}$. The injection volume was $0.2 \mu \mathrm{l}$.

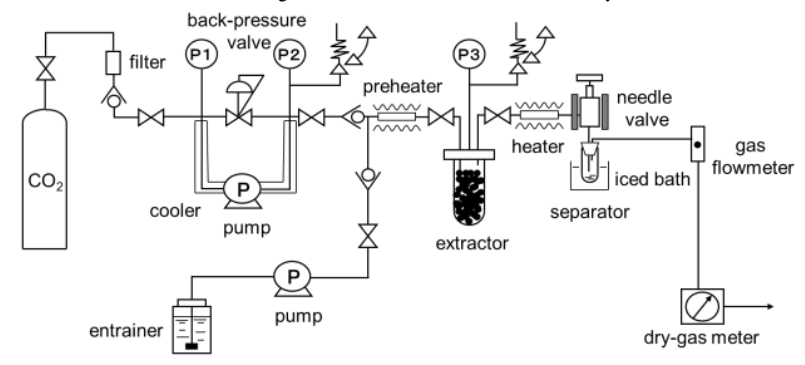

Figure 1. Schematic diagram of supercritical fluid extraction apparatus

\subsection{Measurement of Absorbance Spectra by UV-VIS Spectrophotometer}

The physical property, especially with regards to the purity of the obtained oil, was determined and compared with the commercial virgin coconut oil by measuring the absorbance spectra using a UV-VIS recording spectrophotometer (Shimadzu UV-2400PC). 


\section{Results and Discussion}

\subsection{Drying of Samples}

The comparison between the microwave-assisted and conventional hot-air drying methods of coconut is shown in Figure 2. In the conventional hot air-drying method, it took about $10 \mathrm{~h}$ to completely remove the moisture content, which is about $50 \%$ of the weight of fresh coconut meat. With microwave irradiation, the drying time reduced to about half. In terms of efficiency, these two methods are much better than the freeze-drying method which usually requires about 3 days for the complete removal of the moisture contents.

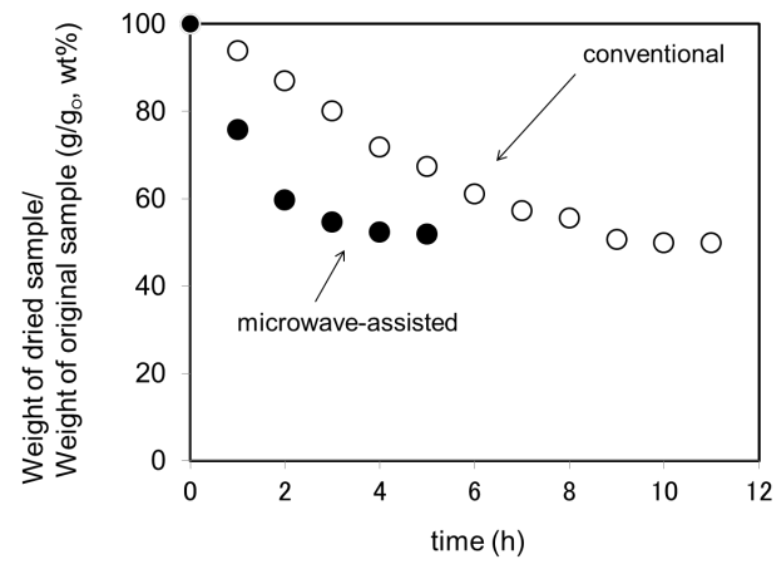

Figure 2. Comparison between conventional and microwave-assisted hot-air drying methods of coconut at $40^{\circ} \mathrm{C}$

\subsection{Preliminary Experiments on Supercritical Carbon Dioxide Extraction}

Preliminary experiments were carried out on various dried coconut samples with different sizes and drying methods, and the results are shown in Figure 3. The recovery was significantly lower using coconut bits (B), which were in sizes of small cubes, as compared to the grated (GT) or ground (GD) samples. The most likely reason for this is that carbon dioxide could not easily penetrate and diffuse through the matrices of the coconut bits. The highest recovery was obtained using the microwave-assisted air-dried samples, thus the microwave-assisted dried grated (GT) samples were used in the succeeding experiments, unless otherwise specified.

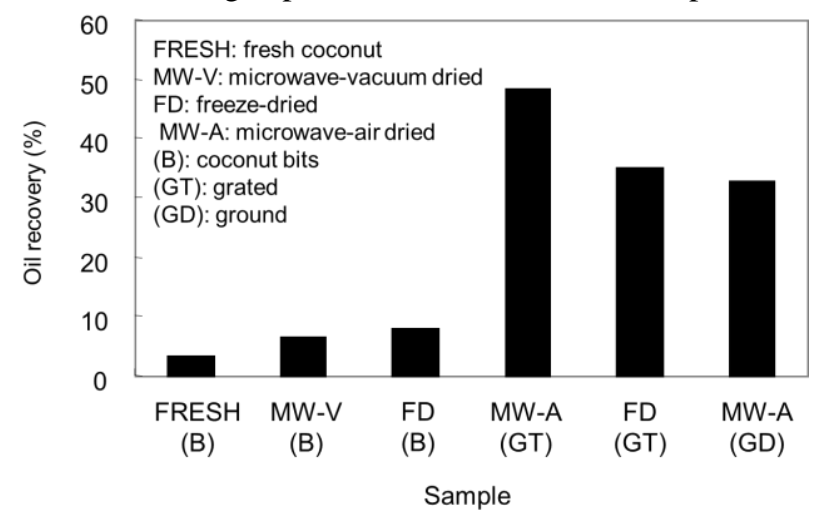

Figure 3. Results of preliminary experiments on supercritical carbon dioxide extraction of coconut oil at $40^{\circ} \mathrm{C}, 20 \mathrm{MPa}$
Supercritical carbon dioxide extraction was then performed until most of the oil in the samples was recovered. As shown in Figure 4, it took less than $20 \mathrm{~h}$ to recover more than $95 \%$ of the oil. This is more advantageous than the conventional cold-press method which requires 48 to $72 \mathrm{~h}$ of processing time to obtain an oil recovery of only about 30 to $50 \%$.

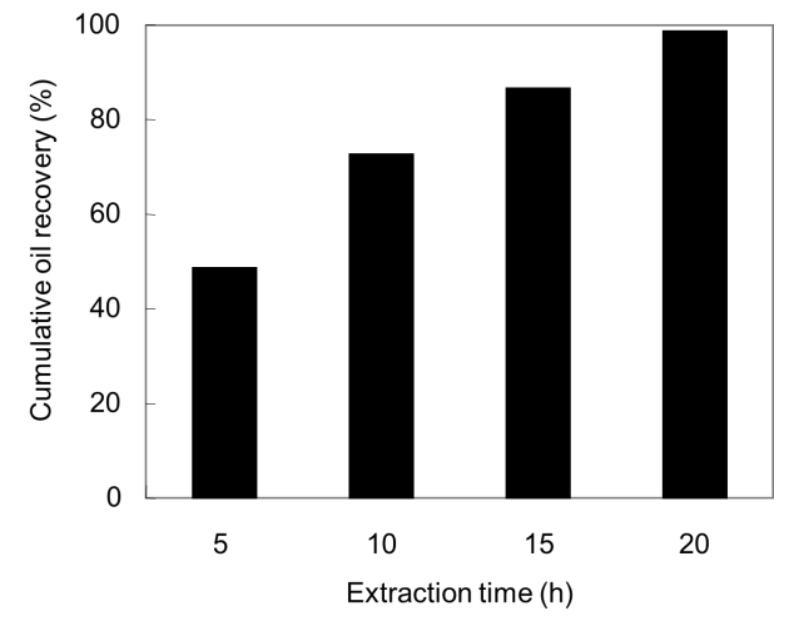

Figure 4. Time course of cumulative recovery of the oil at $40^{\circ} \mathrm{C}$ and $20 \mathrm{MPa}$

\subsection{Physical and Chemical Properties of the Extracts Compared to Commercial Virgin Coconut Oils}

The composition of the obtained oil was analyzed using HPLC and the chromatographs are shown in Figure 5 in comparison with those of the commercial virgin coconut oils and an ordinary coconut oil purchased from Nacalai Tesque (Japan). The chromatograph showing the composition of the obtained oil resembles those of the commercial virgin coconut oils. The fatty acid contents of the oil were then analyzed by GC-FID. The results in Table 1 show that the lauric acid content of the oil obtained using supercritical carbon dioxide is a little bit higher compared to the commercial virgin coconut oils. In addition, the clarity of the extracts is almost identical to the commercial virgin coconut oils based on the measured absorbance spectra as shown in Figure 6.

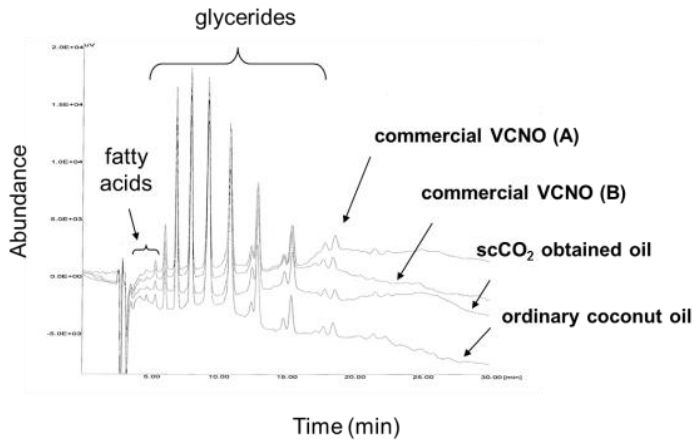

Figure 5. HPLC-RI chromatograms showing chemical composition of the extracted oil in comparison with the two commercial virgin coconut oils and an ordinary coconut oil

The results indicated that the obtained oil has physical and chemical properties that are almost identical to or 
better than the commercial VCNO, thus deemed to possess the same antimicrobial and nutritional properties.

\subsection{Optimization Analyses}

\subsubsection{Effect of Drying Methods on Extraction}

Extraction experiments were carried out on samples dried using 3 different methods, i.e. hot-air, MW-assisted hot-air and freeze-dry methods. As shown by the results in Figure 7, no significant effect of the drying methods on extraction yield was observed. However, the use of microwave irradiation may have an effect on other thermally labile and light-sensitive vitamins and minerals present in the oil. In this regard, the contents of vitamin E on each sample were measured using HPLC but the amount was below the detection limit of the apparatus, thus no valid conclusion could be drawn at present on the effects of the drying methods on the composition of vitamins and minerals present in the extracts.

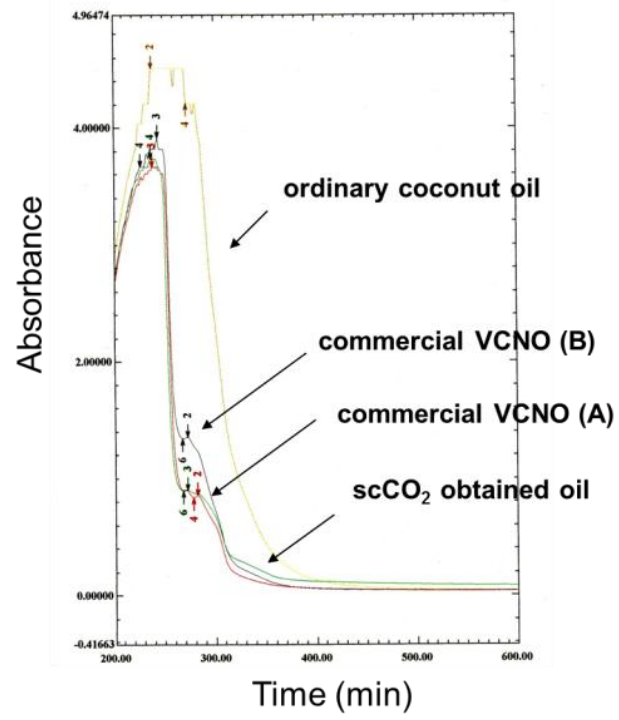

Figure 6. Absorbance spectra indicating the clarity of the extracts in comparison with the commercial virgin coconut oil and an ordinary coconut oil

Table 1. Fatty acid content of the oil in \% relative mass

\begin{tabular}{|c|c|c|c|c|}
\hline Fatty acids & VCNO A & VCNO B & $\mathrm{ScCO}_{2}$ & Ordinary \\
\hline Capric Acid & 7.13 & 7.81 & 7.97 & 7.47 \\
\hline Lauric Acid & 53.20 & 53.89 & 55.96 & 53.10 \\
\hline Myrisric Acid & 21.02 & 19.56 & 19.61 & 20.10 \\
\hline Palmitic Acid & 9.70 & 8.91 & 8.05 & 9.56 \\
\hline Steatic Acid & 2.87 & 2.41 & 2.26 & 2.91 \\
\hline Oleic Acid & 5.29 & 6.25 & 4.68 & 6.87 \\
\hline Lonileic Acid & 0.79 & 1.18 & 1.47 & trace \\
\hline
\end{tabular}

\subsubsection{Effect of Pressure and Addition of EtOH}

At constant temperature of $40^{\circ} \mathrm{C}$, an increase in pressure from 20 to $30 \mathrm{MPa}$ decreased the oil recovery as shown in Figure 8. This was likely due to a decrease in solubility of the coconut oil in supercritical carbon dioxide at higher pressures. In reference to the data reported by King and Bott [18], at constant temperature, the solubility of soybean oil in supercritical carbon dioxide decreases with increasing pressure. At a lower pressure of $10 \mathrm{MPa}$, the yield also decreased significantly. Moreover, the recovery at a temperature of $30^{\circ} \mathrm{C}$ and a pressure of $7 \mathrm{MPa}$ was very low at $0.41 \%$.
Only a slight increase in the yield was observed with the addition of $5 \mathrm{~mol} \% \mathrm{EtOH}$, as entrainer. It is not also recommended to use an entrainer such as $\mathrm{EtOH}$, because the required post-treatment procedures to separate the $\mathrm{EtOH}$ from the extracts would add up to the operating costs.

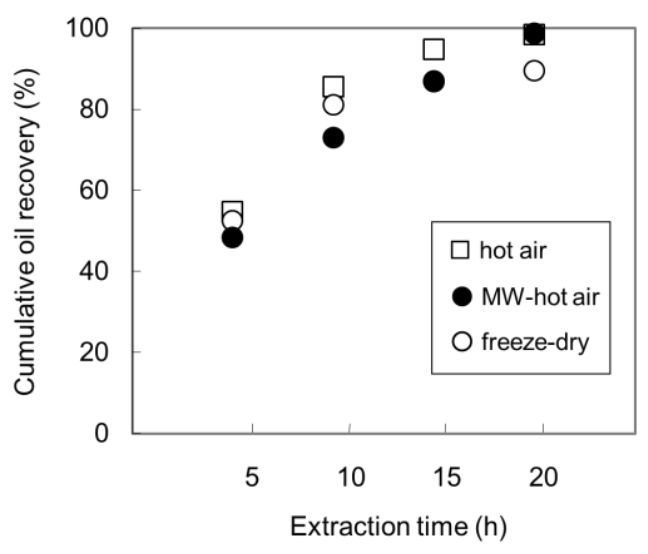

Figure 7. Effect of drying methods on the recovery at $40^{\circ} \mathrm{C}$ and $20 \mathrm{MPa}$

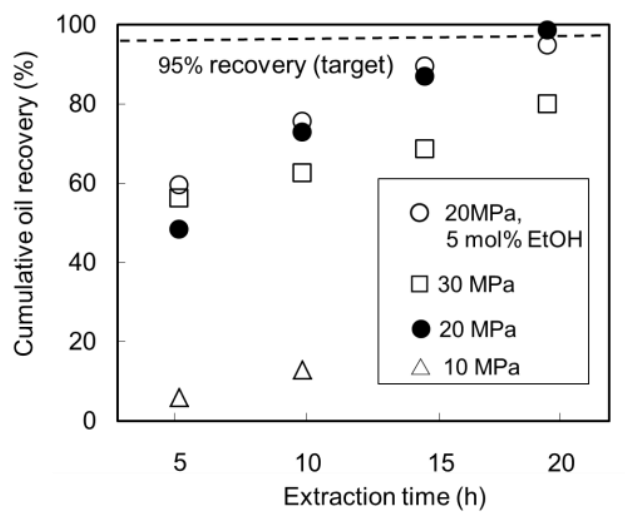

Figure 8. Effect of pressure and addition of EtOH on the recovery of coconut oil at $40^{\circ} \mathrm{C}$

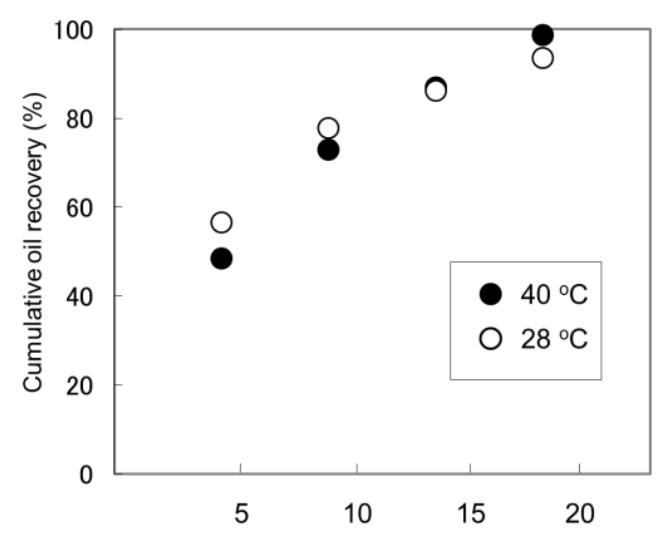

Figure 9. Comparison of extraction at temperatures of 28 and $40^{\circ} \mathrm{C}$ at $20 \mathrm{MPa}$

\subsubsection{Effect of Temperature}

In tropical countries, virgin coconut oil is usually obtained by cold-press method at atmospheric temperature range of 28 to $35^{\circ} \mathrm{C}$. Supercritical carbon dioxide extraction was also carried out at $28^{\circ} \mathrm{C}$, below the critical 
temperature of $\mathrm{CO}_{2}$, at a pressure of $20 \mathrm{MPa}$, and the results in comparison with that at $40^{\circ} \mathrm{C}$ are shown in Figure 9. The initial rate of extraction was relatively higher at subcritical temperature of $28^{\circ} \mathrm{C}$ compared to that at $40^{\circ} \mathrm{C}$, but as extraction time progressed beyond $15 \mathrm{~h}$, the rate decreased. These results suggest that supercritical condition of temperatures would be required for the $\mathrm{CO}_{2}$ to easily penetrate into the sample matrices and for the oil to easily diffuse through it, thus obtaining higher recovery rate especially at low oil contents.

\subsubsection{Effect of Spent $\mathrm{scCO}_{2}$-to-sample Ratio}

At $40^{\circ} \mathrm{C}, 20 \mathrm{MPa}$ and a constant $\mathrm{CO}_{2}$ flowrate of $2 \mathrm{~L} / \mathrm{min}$, the amount of charged coconut sample was varied at 80,55 and $35 \mathrm{~g}$. Results showed that the recovery increases with increasing amount of spent $\mathrm{CO}_{2}$ in relation to the amount of sample charged. However, in commercial plant, $\mathrm{CO}_{2}$ is usually recycled. Thus, larger amount of spent $\mathrm{CO}_{2}$ leads to higher energy cost for recycling of $\mathrm{CO}_{2}$. It was also observed from the results of the experiments that an average of about $25 \mathrm{~g}$ of oil could be obtained from 70 to $80 \mathrm{~g}$ of sample in $5 \mathrm{~h}$. At this condition, about $38 \mathrm{~g}$ of sample should be charged to the extractor to obtain $25 \mathrm{~g}$ of oil at $100 \%$ recovery.

\section{Conclusion}

The application of supercritical carbon dioxide to the extraction of virgin coconut oil was investigated. The sample was pre-dried by microwave-assisted low temperature air drying at $40^{\circ} \mathrm{C}$. The results showed that using supercritical carbon dioxide extraction, an oil recovery of more than $95 \%$ could be obtained at a processing time less than $20 \mathrm{~h}$ compared to the conventional cold-press method which has a normal oil recovery of about 30 to $50 \%$ at a longer processing time of 48 to $72 \mathrm{~h}$. The physical and chemical properties of the extracted oil were almost identical to or better than the commercial VCNO, thus deemed to possess the same antimicrobial and nutritional properties. Extraction conditions were also varied for optimization analyses.

The results of this work add to the many practical applications of supercritical carbon dioxide to the extraction of useful compounds from natural products. The commercialization of the process is expected in the near future to meet the increasing demand for high-quality and high-grade VCNO.

\section{Acknowledgement}

